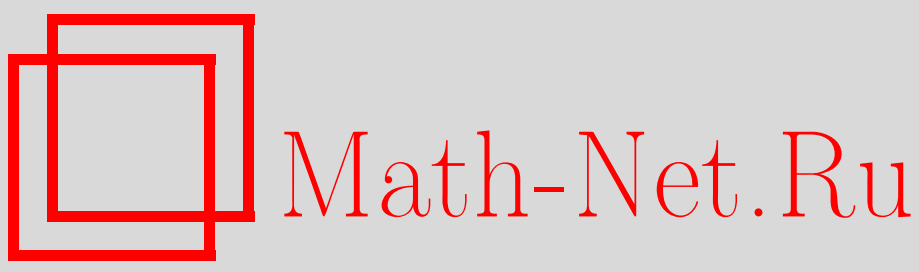

А. Е. Миронов, Об одном кольце коммутирующих дифференциальных операторов ранга два, отвечающем кривой рода два, Матем. сб., 2004, том 195, номер 5, 103114

DOI: https://doi.org/10.4213/sm823

Использование Общероссийского математического портала Math-Net.Ru подразумевает, что вы прочитали и согласны с пользовательским соглашением

http: //www.mathnet.ru/rus/agreement

Параметры загрузки:

IP: 54.197 .217 .227

26 апреля 2023 г., 15:00:12 
УДК 517.43

\author{
А.Е. Миронов
}

\title{
Об одном кольце коммутирующих дифференциальных операторов ранга два, отвечающем кривой рода два
}

\begin{abstract}
В статье предложен метод построения коммутирующих обыкновенных линейных дифференциальных операторов ранга два, отвечающих кривой рода два, а также указан пример таких операторов с полиномиальньми коэффициентами. Библиография: 13 названий.
\end{abstract}

\section{§1. Введение и основные результаты}

В настоящей статье предложен метод нахождения некоторых коммутирующих обыкновенных линейных дифференциальных операторов ранга два, отвечающих кривой рода два.

Уравнения коммутации двух операторов

$$
L_{1}=\frac{d^{n}}{d x^{n}}+\sum_{i=0}^{n-2} u_{i}(x) \frac{d^{i}}{d x^{i}}, \quad L_{2}=\frac{d^{m}}{d x^{m}}+\sum_{i=0}^{m-1} v_{i}(x) \frac{d^{i}}{d x^{i}}, \quad n<m
$$

представляют собой сложную систему нелинейных уравнений на коэффициенты $u_{i}, v_{j}$. Первые результаты по этим уравнениям были получены в 1920-30-е годы Берчналлом и Чаунди [1]-[3]. В частности, Берчналл и Чаунди доказали следующее утверждение.

ЛЕмМа 1. Если $L_{1} L_{2}=L_{2} L_{1}$, то существует ненулевой полином $Q$ от двух коммутирующих переменных такой, что $Q\left(L_{1}, L_{2}\right)=0$.

Например, несложно убедиться, что операторы

$$
L_{1}=\frac{d^{2}}{d x^{2}}-\frac{2}{x^{2}}, \quad L_{2}=\frac{d^{3}}{d x^{3}}-\frac{3}{x^{2}} \frac{d}{d x}+\frac{3}{x^{3}}
$$

коммутируют между собой, при этом они связаны полиномиальным соотношением $L_{1}^{3}=L_{2}^{2}$.

Новый интерес к коммутирующим дифференциальным операторам появился в 1970-е годы в связи с тем, что было замечено, что некоторые нелинейные уравнения типа уравнения Кортевега-де Фриза эквивалентны условию коммутации специально подобранных операторов. Например, операторы

$$
L=\partial_{x}^{2}-u, \quad A=\partial_{t}-\partial_{x}^{3}+\frac{3}{2} u \partial_{x}+\frac{3}{4} u_{x}
$$

Работа выполнена при финансовой поддержке Российского фонда фундаменталњных исследований (гранты №№ 03-01-00403 и 03-01-06482) и Программы поддержки ведущих научных шккол РФ (грант № НШ-2185.2003.1).

(C) А.Е. Миронов 2004 
коммутируют в том и только том случае, когда функция $u(x, t)$ удовлетворяет уравнению Кортевега-де Фриза

$$
u_{t}=\frac{1}{4}\left(6 u u_{x}+u_{x x x}\right)
$$

Наличие $(L, A)$-пары позволяет строить точные решения этих уравнений.

В основе построения операторов $L_{1}$ и $L_{2}$ лежит спектральная кривая $\Gamma$ - пополнение кривой, заданной в $\mathbb{C}^{2}$ уравнением $Q(z, w)=0$. Мы будем рассматривать только случай общего положения, когда кривая Г гладкая. Для каждой точки $P \in \Gamma$ найдется совместная собственная функция $\psi(x, P)$ операторов $L_{1}$ и $L_{2}$. Эта функция имеет существенную особенность в некоторой точке $q \in \Gamma$, а на $\Gamma-\{q\}$ она мероморфна. При этом

$$
L_{i} \psi=\lambda_{i}(P) \psi
$$

где $\lambda_{i}(P)$ - некоторая мероморфная функция на $\Gamma$ с единственным полюсом в точке $q$. Число $l$ линейно независимых совместных собственных функций, отвечающих общей точке $P \in \Gamma$, называется рангом пары $L_{1}, L_{2}$. Порядки операторов $L_{1}$ и $L_{2}$ делятся на $l: n=n^{\prime} l, m=m^{\prime} l$. Порядки полюсов функций $\lambda_{1}$ и $\lambda_{2}$ в точке $q$ равны соответственно $n^{\prime}$ и $m^{\prime}$. В случае ранга $1 \psi$ можно выразить через тэта-функцию кривой Г и коэффициенты операторов восстанавливаются по $\psi$ (см. [4]). В случае ранга $l>1$, как показал Кричевер [5], нахождение $\psi$ сводится к решению интегрального уравнения, точное решение которого получить не удается. На самом деле для нахождения операторов ранга $l>1$ не обязательно знать $\psi$. Кричевер и Новиков [6], [7] предложили следующий метод нахождения таких операторов.

Пусть $\psi_{0}(x, P), \ldots, \psi_{l-1}(x, P), P \in \Gamma,-$ совместные собственные функции, нормированные следующим образом:

$$
\frac{d^{i}}{d x^{i}} \psi_{j}\left(x_{0}, P\right)=\delta_{i j}
$$

где $x_{0}-$ некоторая фиксированная точка. Обозначим через $\Phi$ матрицу Вронского функций $\psi_{j}$

$$
\Phi=\left(\begin{array}{ccc}
\psi_{0} & \ldots & \psi_{l-1} \\
\psi_{0}^{\prime} & \ldots & \psi_{l-1}^{\prime} \\
\ldots \ldots & \ldots & \ldots \ldots \\
\psi_{0}^{(l-1)} & \ldots & \psi_{l-1}^{(l-1)}
\end{array}\right)
$$

Компоненты $\chi_{i}(x, P)$ матрицы

$$
\frac{d \Phi}{d x} \Phi^{-1}=\left(\begin{array}{ccccc}
0 & 1 & 0 & \ldots & 0 \\
0 & 0 & 1 & \ldots & 0 \\
\ldots & \ldots & \ldots & \ldots & \ldots \\
0 & 0 & 0 & \ldots & 1 \\
\chi_{0} & \chi_{1} & \chi_{2} & \ldots & \chi_{l-1}
\end{array}\right)
$$

не зависят от выбора точки $x_{0}$. По функциям $\chi_{i}$ из разложения

$$
\frac{d^{l}}{d x^{l}} \psi_{j}=\chi_{l-1} \frac{d^{l-1}}{d x^{l-1}} \psi_{j}+\cdots+\chi_{0} \psi_{j}
$$


и нормировки (2) можно находить производные $\psi_{j}$ любого поря дка в точке $x_{0}$. Далее, из (1) находятся значения коэффициентов операторов $L_{i}$ в точке $x_{0}$. Поскольку функции $\chi_{j}$ не зависят от $x_{0}$, получаем значения коэффициентов операторов в любой точке. Таким образом, нахождение операторов сводится к нахождению функций $\chi_{j}$. Новиков и Кричевер [6], [7] нашли $\chi_{j}$ в случае кривой рода 1 и ранга 2. Мохов [8], используя подход Новикова и Кричевера, нашел $\chi_{j}$ в случае $g=1$ и $l=3$ (см. также [9]). Как указали Новиков и Гриневич, спектральная теория периодических операторов ранга $l>1$, связанных с кривой $\Gamma$, сводится к спектральной теории операторов ранга 1 после перехода к $l$-листному накрытию кривой $\Gamma[10],[11]$.

Необходимо отметить, что к задаче отыскания пар коммутирующих операторов существует и чисто алгебраический подход. Так, например, Диксмње [12], используя алгебры Вейля, нашел пары коммутирующих операторов рода 1 с полиномиальными коэффициентами.

Основной результат этой работы заключается в следуюшем. Пусть $l=2$ и кривая Г рода 2 задается в $\mathbb{C}^{2}$ уравнением

$$
w^{2}=F(z)=z^{6}+c_{5} z^{5}+c_{4} z^{4}+c_{3} z^{3}+c_{2} z^{2}+c_{1} z+c_{0}
$$

где $c_{0} \neq 0$. Кривая Г допускает голоморфную инволюцию

$$
\sigma: \Gamma \rightarrow \Gamma, \quad \sigma(z, w)=(z,-w)
$$

которая имеет шесть неподвижных точек $\left(z_{i}, 0\right)$, где $z_{i}$ - точки ветвления (корни уравнения $F(z)=0$ ). Мы рассматриваем случай, когда $\sigma \chi_{1}=\chi_{1}$. Тогда функция $\chi_{1}$ определена на $\Gamma / \sigma$, т.е. можно считать, что $\chi_{1}$ зависит только от $z$ и не зависит от $w$. Функцию $\chi_{0}$ мы представляем в виде $\frac{1}{2}\left(\chi_{0}+\sigma \chi_{0}\right)+\frac{1}{2} \sqrt{\left(\chi_{0}-\sigma \chi_{0}\right)^{2}}$, где $\chi_{0}+\sigma \chi_{0}$ и $\left(\chi_{0}-\sigma \chi_{0}\right)^{2}$ также определены на $\mathbb{C}$. Таким образом, мы сводим задачу нахождения $\chi_{i}$ на $\Gamma$ к нахождению трех функций на $\mathbb{C}: \chi_{1}, \chi_{0}+\sigma \chi_{0}$ и $\left(\chi_{0}-\sigma \chi_{0}\right)^{2}$. Смена знака у $\sqrt{\left(\chi_{0}-\sigma \chi_{0}\right)^{2}}$ дает функцию $\sigma \chi_{0}$. Имеет место

Теорема 1. Компоненты $\chi_{0}(x, P)$ и $\chi_{1}(x, P)$ матрицы $\frac{d \Phi}{d x} \Phi^{-1}$ имеют следующий вид:

$$
\begin{aligned}
\chi_{1}(x, P)= & -\frac{\gamma_{1}^{\prime}(x)}{z-\gamma_{1}(x)}-\frac{\gamma_{2}^{\prime}(x)}{z-\gamma_{2}(x)}-\frac{\gamma_{1}^{\prime}(x)}{\gamma_{1}(x)}-\frac{\gamma_{2}^{\prime}(x)}{\gamma_{2}(x)} \\
\chi_{0}(x, P)= & -\frac{1}{2} \frac{H(x) \gamma_{1}^{\prime}(x)}{z-\gamma_{1}(x)}-\frac{1}{2} \frac{H(x) \gamma_{2}^{\prime}(x)}{z-\gamma_{2}(x)}+\frac{1}{2 z}+\frac{\varkappa_{2}(x)}{2}+\frac{1}{2} \sqrt{R(x, z)}, \\
R(x, z)= & \frac{F_{1}(x) \gamma_{1}^{\prime 2}(x)}{\left(z-\gamma_{1}(x)\right)^{2}}+\frac{2 G_{1}(x) \gamma_{1}^{\prime}(x)}{z-\gamma_{1}(x)}+\frac{F_{2}(x) \gamma_{2}^{\prime 2}(x)}{\left(z-\gamma_{2}(x)\right)^{2}}+\frac{2 G_{2}(x) \gamma_{2}^{\prime}(x)}{z-\gamma_{2}(x)} \\
& \quad+\frac{1}{z^{2}}+\frac{1}{z}\left(\frac{2\left(\gamma_{1}(x)+\gamma_{2}(x)\right)}{\gamma_{1}(x) \gamma_{2}(x)}+\frac{c_{1}}{c_{0}}\right)+\frac{\gamma_{1}^{2}(x) \gamma_{2}^{2}(x)}{c_{0}} .
\end{aligned}
$$

Все нули функиии $R$ имеют первый порядок и расположены в точках ветвления, все полюсы имеют второй порядок (поэтому функция $\sqrt{R}$ корректно 
определена на Г). Функции $\gamma_{1}(x)$ и $\gamma_{2}(x)$ удовлетворяют нелинейному дифференциальному уравнению, которое интегрируемо в квадратурах относительно $\gamma_{1}(x)$, если положить

$$
\gamma_{2}(x)=\frac{C-B \gamma_{1}(x)}{B+A \gamma_{1}(x)}, \quad A, B, C \in \mathbb{C} .
$$

В этом случае решение этого уравнения имеет следующий вид:

$$
\gamma_{1}^{-1}\left(y_{1}\right)=\int \sqrt{\frac{y_{1} y_{2}}{2 c_{0}\left(y_{2}-y_{1}\right)^{3}}\left(y_{2}^{2} F\left(y_{1}\right)-\frac{y_{1}^{2} F\left(y_{2}\right)\left(B+A y_{1}\right)^{4}}{\left(B^{2}+A C\right)^{2}}\right)} d y_{1},
$$

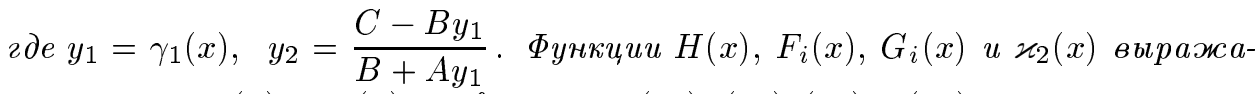
ются через $\gamma_{1}(x)$ и $\gamma_{2}(x)$ по формулам (24), (20)-(23) и (14).

СлеДСтвиЕ 1. Оператор, отвечающий функщии

$$
\lambda=\frac{1}{2 z^{3}}+\frac{w}{2 z^{3} \sqrt{c_{0}}}+\frac{c_{1}}{4 z^{2} c_{0}}+\frac{1}{z}\left(\frac{c_{2}}{4 c_{0}}-\frac{c_{1}^{2}}{16 c_{0}^{2}}\right)
$$

имеет вид

$$
L=\frac{d^{6}}{d x^{6}}+f_{4} \frac{d^{4}}{d x^{4}}+f_{3} \frac{d^{3}}{d x^{3}}+f_{2} \frac{d^{2}}{d x^{2}}+f_{1} \frac{d}{d x}+f_{0}
$$

əдe

$$
\begin{aligned}
& f_{4}= \alpha-3 a_{0}, \quad f_{3}=-6 a_{0}^{\prime}-3 b_{1}, \\
& f_{2}= \beta-7 a_{0}^{\prime \prime}-6 b_{1}^{\prime}-3 a_{1}-2 \alpha a_{0}+3 a_{0}^{2}, \\
& f_{1}=-2 \alpha b_{1}+3 a_{0} b_{1}-3 b_{2}-2 \alpha a_{0}^{\prime}+6 a_{0} a_{0}^{\prime}-6 a_{1}^{\prime}-7 b_{1}^{\prime \prime}-4 a_{0}^{\prime \prime \prime}, \\
& f_{0}=\gamma-\beta a_{0}+\alpha a_{0}^{2}-a_{0}^{3}-2 \alpha a_{1}+3 a_{0} a_{1}+2\left(a_{0}^{\prime}\right)^{2} \\
& \quad \quad-2 \alpha b_{1}^{\prime}-6 b_{2}^{\prime}-\alpha a_{0}^{\prime \prime}+3 a_{0} a_{0}^{\prime \prime}-7 a_{1}^{\prime \prime}-4 b_{1}^{\prime \prime \prime}-a_{0}^{\mathrm{IV}} .
\end{aligned}
$$

Функции $a_{0}(x), a_{1}(x), b_{1}(x)$ и $b_{2}(x)$ находятся из разложсений $\chi_{0}$ и $\chi_{1}$ в ряд по степеням $z$ :

$$
\begin{aligned}
\chi_{0}= & \frac{1}{z}+a_{0}+a_{1} z+O\left(z^{2}\right), \quad \chi_{1}=b_{1} z+b_{2} z^{2}+O\left(z^{3}\right), \\
a_{0}(x)= & \frac{H(x) \gamma_{1}^{\prime}(x)}{2 \gamma_{1}(x)}+\frac{H(x) \gamma_{2}^{\prime}(x)}{2 \gamma_{2}(x)}+\frac{\varkappa_{2}}{2}+\frac{\gamma_{1}(x)+\gamma_{2}(x)}{2 \gamma_{1}(x) \gamma_{2}(x)}+\frac{c_{1}}{4 c_{0}}, \\
a_{1}(x)= & \frac{H(x) \gamma_{1}^{\prime}(x)}{2 \gamma_{1}^{2}(x)}+\frac{H(x) \gamma_{2}^{\prime}(x)}{2 \gamma_{2}^{2}(x)}+\frac{F_{1}(x) \gamma_{1}^{\prime 2}(x)}{4 \gamma_{1}^{2}(x)}-\frac{G_{1}(x) \gamma_{1}^{\prime}(x)}{2 \gamma_{1}(x)}+\frac{F_{2}(x) \gamma_{2}^{\prime 2}(x)}{4 \gamma_{2}^{2}(x)} \\
& \quad-\frac{G_{2}(x) \gamma_{2}^{\prime}(x)}{2 \gamma_{2}(x)}+\frac{\gamma_{1}^{2}(x) \gamma_{2}^{2}(x)}{4 c_{0}}-\frac{1}{16}\left(\frac{2\left(\gamma_{1}(x)+\gamma_{2}(x)\right)}{\gamma_{1}(x) \gamma_{2}(x)}+\frac{c_{1}}{c_{0}}\right)^{2}, \\
b_{1}(x)= & \frac{\gamma_{1}^{\prime}(x)}{\gamma_{1}^{2}(x)}+\frac{\gamma_{2}^{\prime}(x)}{\gamma_{2}^{2}(x)}, \quad b_{2}(x)=\frac{\gamma_{1}^{\prime}(x)}{\gamma_{1}^{3}(x)}+\frac{\gamma_{2}^{\prime}(x)}{\gamma_{2}^{3}(x)},
\end{aligned}
$$


$\alpha, \beta$ и -коэффициенты разложения $\lambda$ в ряд

$$
\begin{aligned}
\lambda & =\frac{1}{z^{3}}+\frac{\alpha}{z^{2}}+\frac{\beta}{z}+\gamma+O(z), \\
\alpha=\frac{c_{1}}{2 c_{0}}, \quad \beta & =\frac{c_{2}}{2 c_{0}}-\frac{c_{1}^{2}}{8 c_{0}^{2}}, \quad \gamma=\frac{c_{1}^{3}}{32 c_{0}^{3}}-\frac{c_{1} c_{2}}{8 c_{0}^{2}}+\frac{c_{3}}{4 c_{0}} .
\end{aligned}
$$

Операторы, коммутирующие с $L$, легко находятся из уравнений коммутации.

Отметим, что при $c_{1}=c_{5}=0, c_{3}=8 c_{0}$ и при

$$
\gamma_{1}(x)=-\gamma_{2}(x)=\frac{1}{x}
$$

коэффициенты операторов являются рациональными функциями. Среди таких операторов можно выделить операторы с полиномиальными коэффициентами. Укажем пример. Пусть кривая Г задана уравнением

$$
w^{2}=z^{6}+2 z^{3}+\frac{1}{4}
$$

Тогда операторы

$$
\begin{aligned}
L_{1}= & \partial_{x}^{6}-\frac{3 x^{4}}{16} \partial_{x}^{4}-\frac{3 x^{3}}{2} \partial_{x}^{3}+\frac{3}{256}\left(x^{8}-576 x^{2}\right) \partial_{x}^{2}+\left(\frac{3 x^{7}}{32}-6 x\right) \partial_{x} \\
& -\frac{x^{12}}{4096}+\frac{23 x^{6}}{64}-\frac{3}{2}, \\
L_{2}= & \partial_{x}^{8}-\frac{x^{4}}{4} \partial_{x}^{6}-3 x^{3} \partial_{x}^{5}+\frac{1}{128}\left(3 x^{8}-2368 x^{2}\right) \partial_{x}^{4}+\frac{1}{8}\left(3 x^{7}-320 x\right) \partial_{x}^{3} \\
& -\left(\frac{x^{12}}{1024}-\frac{45 x^{6}}{16}+30\right) \partial_{x}^{2}-\left(\frac{3 x^{11}}{256}-\frac{35 x^{5}}{4}\right) \partial_{x} \\
& +\frac{x^{16}-3712 x^{10}+716800 x^{4}}{65536}, \\
L_{3}= & \partial_{x}^{10}-\frac{5 x^{4}}{16} \partial_{x}^{8}-5 x^{3} \partial_{x}^{7}+\frac{5}{128}\left(x^{8}-1024 x^{2}\right) \partial_{x}^{6}+\frac{5}{16}\left(3 x^{7}-416 x\right) \partial_{x}^{5} \\
& -\left(\frac{5 x^{12}}{2048}-\frac{85 x^{6}}{8}+150\right) \partial_{x}^{4}-\frac{5}{256}\left(3 x^{11}-3136 x^{5}\right) \partial_{x}^{3} \\
& +\frac{5\left(x^{16}-8192 x^{10}+2437120 x^{4}\right)}{65536} \partial_{x}^{2}+\frac{5}{4096}\left(x^{15}-2464 x^{9}+215040 x^{3}\right) \partial_{x} \\
& -\frac{x^{20}}{1048576}+\frac{15 x^{14}}{2048}-\frac{1505 x^{8}}{256}+\frac{525 x^{2}}{4}
\end{aligned}
$$

попарно коммутируют. Операторы $L_{1}, L_{2}$ и $L_{3}$ задают коммутативное кольцо дифоференциальных операторов, изоморфное кольцу мероморфных функций на Г с полюсом в точке $q$. Между $L_{1}$ и $L_{2}$ справедливо следующее алгебраическое соотношение:

$$
L_{2}^{3}=L_{1}^{4}-4 L_{1}^{3}+3 L_{1}^{2}
$$


Через $\widetilde{\Gamma}$ обозначим кривую Берчналла-Чаунди, соответствуюшую паре операторов $L_{1}$ и $L_{2}$. Кривая $\widetilde{\Gamma}$ имеет каспидальную особую точку $(0,0)$. Операторы $L_{1}$ и $L_{2}$ отвечают функциям

$$
\lambda_{1}=\frac{1}{2 z^{3}}+\frac{w}{z^{3}}+2, \quad \lambda_{2}=\frac{1}{2 z^{4}}+\frac{w}{z^{4}}+\frac{2}{z}
$$

которые задают бирациональную эквивалентность

$$
\pi: \Gamma \rightarrow \widetilde{\Gamma}, \quad \pi(z, w)=\left(\lambda_{1}, \lambda_{2}\right)
$$

Прообразом каспидальной точки служит точка $\sigma(q)$. Для того чтобы отображение $\pi$ являлось морфизмом, необходимо пополнить $\widetilde{\Gamma}$ на бесконечности каспидальной точкой типа $(3,4)$, при этом ее прообразом будет точка $q$.

Автор благодарит И. А. Тайманова за проявленный интерес к работе.

\section{§ 2. Доказательство теоремы 1}

Рассмотрим сначала общий случай кривой рода $g$ и операторов ранга $l$. Совместные собственные функции $\psi_{j}(x, P)$ операторов $L_{1}$ и $L_{2}$ обладают следующими свойствами [5]. На $\Gamma-\{q\}$ функции $\psi_{j}$ имеют $l g$ простых полюсов $P_{1}, \ldots, P_{l g}$, причем $P_{i}$ не зависят от $x$. В окрестности $q$ справедливо следующее асимптотическое разложение:

$$
\psi(x, P)=\left(\sum_{j=0}^{\infty} \xi_{j}(x) k^{-j}\right) \Psi
$$

где $k^{-1}$ - локальньй параметр в окрестности точки $q, \xi_{0}=(1,0, \ldots, 0), \quad \psi=$ $\left(\psi_{0}, \ldots, \psi_{l-1}\right), \Psi$ - решение матричного диффференциального уравнения

$$
A=\left(\begin{array}{cccccc}
\frac{d}{d x} \Psi=A \Psi \\
0 & 1 & 0 & \ldots & 0 & 0 \\
0 & 0 & 1 & \ldots & 0 & 0 \\
\ldots \ldots & \ldots & \ldots & \ldots & \ldots \ldots & \ldots \\
0 & 0 & 0 & \ldots & 0 & 1 \\
k+g_{0}(x) & g_{1}(x) & g_{2}(x) & \ldots & g_{l-2}(x) & 0
\end{array}\right),
$$

$g_{i}(x)$ - некоторые функции. Вычеты $v_{i j}(x)$ функций $\psi_{j}, j<l-1$, в точках $P_{i}$ пропорциональны вычетам $v_{i, l-1}(x)$ функции $\psi_{l-1}$ :

$$
v_{i j}(x)=\alpha_{i j} v_{i, l-1}(x)
$$

Набор $\left(P_{j}, \alpha_{i j}\right)$ называется параметрами Тюрина. Компоненты $\chi_{i}(x, P)$ матрицы $\frac{d \Phi}{d x} \Phi^{-1}$ обладают следующим свойством [5]. Это мероморфные функции на $Г$ 
c $l g$ полюсами $P_{1}(x), \ldots, P_{l g}(x)$, причем при $x=x_{0}$ эти точки совпадают с полюсами $P_{1}, \ldots, P_{l g}$ функций $\psi_{j}$. В окрестности точки $q$ функции $\chi_{i}$ имеют вид:

$$
\begin{aligned}
\chi_{0}(x, P) & =k+g_{0}(x)+O\left(k^{-1}\right), \\
\chi_{j}(x, P) & =g_{j}(x)+O\left(k^{-1}\right), \quad j<l-1, \\
\chi_{l-1}(x, P) & =O\left(k^{-1}\right) .
\end{aligned}
$$

В точках $P_{i}(x)$ справедливы разложения

$$
\chi_{j}=\frac{c_{i j}(x)}{k-\gamma_{i}(x)}+d_{i j}(x)+O\left(k-\gamma_{i}(x)\right) .
$$

Обозначим через $\alpha_{i j}(x)$ отношение $\frac{c_{i j}(x)}{c_{i, l-1}(x)}, 0 \leqslant j \leqslant l-1,1 \leqslant i \leqslant l g$. Следующая теорема доказана Кричевером [5].

Теорема 2. Параметры $\gamma_{i}(x), \alpha_{i j}(x), d_{i j}(x), 0 \leqslant j \leqslant l-2,1 \leqslant i \leqslant l g$, удовлетворяют уравнениям:

$$
\begin{gathered}
c_{i, l-1}(x)=-\gamma_{i}^{\prime}(x) \\
d_{i 0}(x)=\alpha_{i 0}(x) \alpha_{i, l-2}(x)+\alpha_{i 0}(x) d_{i, l-1}(x)-\alpha_{i 0}^{\prime}(x), \\
d_{i j}(x)=\alpha_{i j}(x) \alpha_{i, l-2}(x)-\alpha_{i, j-1}(x)+\alpha_{i j}(x) d_{i, l-1}(x)-\alpha_{i j}^{\prime}(x), \quad j \geqslant 1 .
\end{gathered}
$$

Отметим, что эти уравнения инвариантны относительно выбора локального параметра в окрестности $P_{i}(x)$ и $q$, т.е. при замене локального параметра соответствуюшие коэффициенты разложений будут также удовлетворять этой системе.

При $g=2$ и $l=2$ по теореме 2 в окрестности точки $P_{i}(x), 1 \leqslant i \leqslant 4$, функции $\chi_{i}$ имеют вид:

$$
\begin{aligned}
& \chi_{0}(x, P)=\frac{-\alpha_{i 0}(x) \gamma_{i}^{\prime}(x)}{k-\gamma_{i}(x)}+d_{i 0}(x)+O\left(k-\gamma_{i}(x)\right), \\
& \chi_{1}(x, P)=\frac{-\gamma_{i}^{\prime}(x)}{k-\gamma_{i}(x)}+d_{i 1}(x)+O\left(k-\gamma_{i}(x)\right),
\end{aligned}
$$

причем имеет место равенство

$$
d_{i 0}(x)=\alpha_{i 0}^{2}(x)+\alpha_{i 0}(x) d_{i 1}(x)-\alpha_{i 0}^{\prime}(x),
$$

а в окрестности $q-$ следующий вид:

$$
\begin{aligned}
& \chi_{0}(x, P)=k+g_{0}(x)+O\left(k^{-1}\right), \\
& \chi_{1}(x, P)=O\left(k^{-1}\right) .
\end{aligned}
$$

Пусть Г задается уравнением (3), а точка $q$ имеет координаты $(0, \sqrt{F(0)})$. Выберем в окрестности $P_{i}(x)$ локальный параметр $z-\gamma_{i}(x)$, а в окрестности $q-$ локальньй параметр $z$. Пусть $\sigma \chi_{1}=\chi_{1}$. Тогда можно считать, что $\sigma P_{1}(x)=P_{3}(x)$ 
и $\sigma P_{2}(x)=P_{4}(x)$, следовательно, $\gamma_{1}=\gamma_{3}, \gamma_{2}=\gamma_{4}, d_{11}=d_{31}, d_{21}=d_{41}$. Таким образом, в силу ( 7 ) имеет место равенство

$$
\chi_{1}=\frac{1}{2}\left(\chi_{1}+\sigma \chi_{1}\right)=\frac{-\gamma_{1}^{\prime}}{z-\gamma_{1}}+\frac{-\gamma_{2}^{\prime}}{z-\gamma_{2}}-\varkappa_{1},
$$

где $\varkappa_{1}(x)$ - некоторая функция.

Так как $\chi_{1}(q)=0$, то $\varkappa_{1}=\gamma_{1}^{\prime} / \gamma_{1}+\gamma_{2}^{\prime} / \gamma_{2}$. Из разложения (7) выводим, что

$$
d_{11}=\frac{-\gamma_{2}^{\prime}}{\gamma_{1}-\gamma_{2}}-\varkappa_{1}, \quad d_{21}=\frac{-\gamma_{1}^{\prime}}{\gamma_{2}-\gamma_{1}}-\varkappa_{1}
$$

Далее, из разложений (6) и (9) получаем

$$
\begin{gathered}
\chi_{0}+\sigma \chi_{0}=-\frac{\left(\alpha_{10}+\alpha_{30}\right) \gamma_{1}^{\prime}}{z-\gamma_{1}}-\frac{\left(\alpha_{20}+\alpha_{40}\right) \gamma_{2}^{\prime}}{z-\gamma_{2}}+\frac{1}{z}+\varkappa_{2}, \\
\left(\chi_{0}-\sigma \chi_{0}\right)^{2}=\frac{\left(\alpha_{30}-\alpha_{10}\right)^{2} \gamma_{1}^{\prime 2}}{\left(z-\gamma_{1}\right)^{2}}+\frac{2\left(d_{10}-d_{30}\right)\left(\alpha_{30}-\alpha_{10}\right) \gamma_{1}^{\prime}}{z-\gamma_{1}} \\
\quad+\frac{\left(\alpha_{40}-\alpha_{20}\right)^{2} \gamma_{2}^{\prime 2}}{\left(z-\gamma_{2}\right)^{2}}+\frac{2\left(d_{20}-d_{40}\right)\left(\alpha_{40}-\alpha_{20}\right) \gamma_{2}^{\prime}}{z-\gamma_{2}}+\frac{1}{z^{2}}+\frac{V}{z}+\varkappa,
\end{gathered}
$$

где $\varkappa(x), \varkappa_{2}(x)$ и $V(x)$ - некоторые функции. Из равенства $(12)$ получаем

$$
\begin{aligned}
& d_{10}+d_{30}=-\frac{\left(\alpha_{20}+\alpha_{40}\right) \gamma_{2}^{\prime}}{\gamma_{1}-\gamma_{2}}+\frac{1}{\gamma_{1}}+\varkappa_{2}, \\
& d_{20}+d_{40}=-\frac{\left(\alpha_{10}+\alpha_{30}\right) \gamma_{1}^{\prime}}{\gamma_{2}-\gamma_{1}}+\frac{1}{\gamma_{2}}+\varkappa_{2},
\end{aligned}
$$

откуда

$$
d_{10}+d_{30}-\left(d_{20}+d_{40}\right)=-\frac{\left(\alpha_{20}+\alpha_{40}\right) \gamma_{2}^{\prime}}{\gamma_{1}-\gamma_{2}}+\frac{\left(\alpha_{10}+\alpha_{30}\right) \gamma_{1}^{\prime}}{\gamma_{2}-\gamma_{1}}+\frac{1}{\gamma_{1}}-\frac{1}{\gamma_{2}} .
$$

Из формулы (13) имеем

$$
\left(\chi_{0}-\sigma \chi_{0}\right)^{2}=\frac{g}{\left(z-\gamma_{1}\right)^{2}\left(z-\gamma_{2}\right)^{2} z^{2}},
$$

где

$$
\begin{aligned}
g= & z^{6} \varkappa+z^{5}\left(V-2 \varkappa\left(\gamma_{1}+\gamma_{2}\right)+2\left(d_{20}-d_{40}\right)\left(\alpha_{40}-\alpha_{20}\right) \gamma_{2}^{\prime}\right. \\
& \left.+2\left(d_{10}-d_{30}\right)\left(\alpha_{30}-\alpha_{10}\right) \gamma_{1}^{\prime}\right)+z^{4}\left(\varkappa\left(\gamma_{1}^{2}+\gamma_{2}^{2}+4 \gamma_{1} \gamma_{2}\right)-2 V\left(\gamma_{1}+\gamma_{2}\right)\right. \\
& -2\left(\gamma_{2}+2 \gamma_{1}\right)\left(d_{20}-d_{40}\right)\left(\alpha_{40}-\alpha_{20}\right) \gamma_{2}^{\prime}-2\left(\gamma_{1}+2 \gamma_{2}\right)\left(d_{10}-d_{30}\right)\left(\alpha_{30}-\alpha_{10}\right) \gamma_{1}^{\prime} \\
& \left.+\left(\alpha_{40}-\alpha_{20}\right)^{2} \gamma_{2}^{\prime 2}+\left(\alpha_{30}-\alpha_{10}\right)^{2} \gamma_{1}^{\prime 2}+1\right) \\
& +z^{3}\left(-2\left(\gamma_{1} \gamma_{2}^{2}+\gamma_{2} \gamma_{1}^{2}\right) \varkappa+V\left(\gamma_{1}^{2}+\gamma_{2}^{2}+4 \gamma_{1} \gamma_{2}\right)-2\left(\gamma_{1}+\gamma_{2}\right)\right. \\
& +2\left(\gamma_{1}^{2}+2 \gamma_{1} \gamma_{2}\right)\left(d_{20}-d_{40}\right)\left(\alpha_{40}-\alpha_{20}\right) \gamma_{2}^{\prime} \\
& +2\left(\gamma_{2}^{2}+2 \gamma_{1} \gamma_{2}\right)\left(d_{10}-d_{30}\right)\left(\alpha_{30}-\alpha_{10}\right) \gamma_{1}^{\prime}-2\left(\alpha_{40}-\alpha_{20}\right)^{2} \gamma_{2}^{\prime 2} \gamma_{1} \\
& \left.-2\left(\alpha_{30}-\alpha_{10}\right)^{2} \gamma_{1}^{\prime 2} \gamma_{2}\right)+z^{2}\left(\varkappa \gamma_{1}^{2} \gamma_{2}^{2}-V 2\left(\gamma_{1} \gamma_{2}^{2}+\gamma_{2} \gamma_{1}^{2}\right)+\left(\gamma_{1}^{2}+\gamma_{2}^{2}+4 \gamma_{1} \gamma_{2}\right)\right. \\
& -2\left(d_{20}-d_{40}\right)\left(\alpha_{40}-\alpha_{20}\right) \gamma_{2}^{\prime} \gamma_{2} \gamma_{1}^{2}-2\left(d_{10}-d_{30}\right)\left(\alpha_{30}-\alpha_{10}\right) \gamma_{1}^{\prime} \gamma_{1} \gamma_{2}^{2} \\
& \left.+\gamma_{1}^{2}\left(\alpha_{40}-\alpha_{20}\right)^{2} \gamma_{2}^{\prime 2}+\gamma_{2}^{2}\left(\alpha_{30}-\alpha_{10}\right)^{2} \gamma_{1}^{\prime 2} \gamma^{2}\right) \\
& +z\left(\gamma_{1}^{2} \gamma_{2}^{2} V-2\left(\gamma_{1} \gamma_{2}^{2}+\gamma_{2} \gamma_{1}^{2}\right)\right)+\gamma_{1}^{2} \gamma_{2}^{2} .
\end{aligned}
$$


Функция $\left(\chi_{0}-\sigma \chi_{0}\right)^{2}$ имеет нули в шести точках ветвления. Так как $\operatorname{deg} g=6$, то нули $g$ совпадают с нулями функции $F(x)$, следовательно, мы имеем равенства

$$
\begin{aligned}
& V-2 \varkappa\left(\gamma_{1}+\gamma_{2}\right)+2\left(d_{20}-d_{40}\right)\left(\alpha_{40}-\alpha_{20}\right) \gamma_{2}^{\prime} \\
& \quad+2\left(d_{10}-d_{30}\right)\left(\alpha_{30}-\alpha_{10}\right) \gamma_{1}^{\prime}-c_{5} \varkappa=0 \\
& \varkappa\left(\gamma_{1}^{2}+\gamma_{2}^{2}+4 \gamma_{1} \gamma_{2}\right)-2 V\left(\gamma_{1}+\gamma_{2}\right)-2\left(\gamma_{2}+2 \gamma_{1}\right)\left(d_{20}-d_{40}\right)\left(\alpha_{40}-\alpha_{20}\right) \gamma_{2}^{\prime} \\
& \quad-2\left(\gamma_{1}+2 \gamma_{2}\right)\left(d_{10}-d_{30}\right)\left(\alpha_{30}-\alpha_{10}\right) \gamma_{1}^{\prime}+\left(\alpha_{40}-\alpha_{20}\right)^{2} \gamma_{2}^{\prime 2} \\
& \quad+\left(\alpha_{30}-\alpha_{10}\right)^{2} \gamma_{1}^{\prime 2}+1-c_{4} \varkappa=0 \\
& -2\left(\gamma_{1} \gamma_{2}^{2}+\gamma_{2} \gamma_{1}^{2}\right) \varkappa+V\left(\gamma_{1}^{2}+\gamma_{2}^{2}+4 \gamma_{1} \gamma_{2}\right)-2\left(\gamma_{1}+\gamma_{2}\right) \\
& \quad+2\left(\gamma_{1}^{2}+2 \gamma_{1} \gamma_{2}\right)\left(d_{20}-d_{40}\right)\left(\alpha_{40}-\alpha_{20}\right) \gamma_{2}^{\prime} \\
& \quad+2\left(\gamma_{2}^{2}+2 \gamma_{1} \gamma_{2}\right)\left(d_{10}-d_{30}\right)\left(\alpha_{30}-\alpha_{10}\right) \gamma_{1}^{\prime} \\
& \quad-2\left(\alpha_{40}-\alpha_{20}\right)^{2} \gamma_{2}^{\prime 2} \gamma_{1}-2\left(\alpha_{30}-\alpha_{10}\right)^{2} \gamma_{1}^{\prime 2} \gamma_{2}-c_{3} \varkappa=0 \\
& \varkappa \gamma_{1}^{2} \gamma_{2}^{2}-V 2\left(\gamma_{1} \gamma_{2}^{2}+\gamma_{2} \gamma_{1}^{2}\right)+\left(\gamma_{1}^{2}+\gamma_{2}^{2}+4 \gamma_{1} \gamma_{2}\right) \\
& \quad-2\left(d_{20}-d_{40}\right)\left(\alpha_{40}-\alpha_{20}\right) \gamma_{2}^{\prime} \gamma_{2} \gamma_{1}^{2}-2\left(d_{10}-d_{30}\right)\left(\alpha_{30}-\alpha_{10}\right) \gamma_{1}^{\prime} \gamma_{1} \gamma_{2}^{2} \\
& \quad+\gamma_{1}^{2}\left(\alpha_{40}-\alpha_{20}\right)^{2} \gamma_{2}^{\prime 2}+\gamma_{2}^{2}\left(\alpha_{30}-\alpha_{10}\right)^{2} \gamma_{1}^{\prime 2} \gamma^{2}-c_{2} \varkappa=0 \\
& \gamma_{1}^{2} \gamma_{2}^{2} V-2\left(\gamma_{1} \gamma_{2}^{2}+\gamma_{2} \gamma_{1}^{2}\right)-c_{1} \varkappa=0 \\
& \gamma_{1}^{2} \gamma_{2}^{2}-c_{0} \varkappa=0 .
\end{aligned}
$$

Из последних двух уравнений получаем

$$
\varkappa=\frac{\gamma_{1}^{2} \gamma_{2}^{2}}{c_{0}}, \quad V=\frac{2\left(\gamma_{1}+\gamma_{2}\right)}{\gamma_{1} \gamma_{2}}+\frac{c_{1}}{c_{0}}
$$

Решим систему уравнений (16)-(19) относительно $\left(\alpha_{30}-\alpha_{10}\right)^{2}, \quad\left(\alpha_{40}-\alpha_{20}\right)^{2}$, $\left(d_{20}-d_{40}\right)\left(\alpha_{40}-\alpha_{20}\right)$ и $\left(d_{10}-d_{30}\right)\left(\alpha_{30}-\alpha_{10}\right)$. Получим

$$
\begin{gathered}
F_{1}=\left(\alpha_{30}-\alpha_{10}\right)^{2}=\frac{\gamma_{2}^{2} F\left(\gamma_{1}\right)}{c_{0} \gamma_{1}^{\prime 2}\left(\gamma_{1}-\gamma_{2}\right)^{2}} \\
F_{2}=\left(\alpha_{40}-\alpha_{20}\right)^{2}=\frac{\gamma_{1}^{2} F\left(\gamma_{2}\right)}{c_{0} \gamma_{2}^{\prime 2}\left(\gamma_{2}-\gamma_{1}\right)^{2}} \\
G_{1}=\left(d_{10}-d_{30}\right)\left(\alpha_{30}-\alpha_{10}\right) \\
=\frac{1}{2\left(\gamma_{2}-\gamma_{1}\right) \gamma_{1}^{\prime}}\left(\frac{\gamma_{1}^{2} F\left(\gamma_{2}\right)}{c_{0}\left(\gamma_{2}-\gamma_{1}\right)^{2}}+\frac{\gamma_{2}^{2} F\left(\gamma_{1}\right)}{c_{0}\left(\gamma_{1}-\gamma_{2}\right)^{2}}-\frac{\gamma_{1}^{2} \gamma_{2}^{2}}{c_{0}}\left(3 \gamma_{1}^{2}+2 \gamma_{1} \gamma_{2}+\gamma_{2}^{2}\right)\right. \\
\left.-\frac{2\left(\gamma_{1}+\gamma_{2}\right)}{\gamma_{1}}-\frac{c_{1} \gamma_{2}}{c_{0}}+1-c_{4} \frac{\gamma_{1}^{2} \gamma_{2}^{2}}{c_{0}}-c_{5} \frac{\gamma_{1}^{2} \gamma_{2}^{2}}{c_{0}}\left(\gamma_{2}+2 \gamma_{1}\right)\right) \\
G_{2}=\left(d_{20}-d_{40}\right)\left(\alpha_{40}-\alpha_{20}\right) \\
=\frac{1}{2\left(\gamma_{1}-\gamma_{2}\right) \gamma_{2}^{\prime}}\left(\frac{\gamma_{1}^{2} F\left(\gamma_{2}\right)}{c_{0}\left(\gamma_{2}-\gamma_{1}\right)^{2}}+\frac{\gamma_{2}^{2} F\left(\gamma_{1}\right)}{c_{0}\left(\gamma_{1}-\gamma_{2}\right)^{2}}-\frac{\gamma_{1}^{2} \gamma_{2}^{2}}{c_{0}}\left(\gamma_{1}^{2}+2 \gamma_{1} \gamma_{2}+3 \gamma_{2}^{2}\right)\right. \\
\left.-\frac{2\left(\gamma_{1}+\gamma_{2}\right)}{\gamma_{2}}-\frac{c_{1} \gamma_{1}}{c_{0}}+1-c_{4} \frac{\gamma_{1}^{2} \gamma_{2}^{2}}{c_{0}}-c_{5} \frac{\gamma_{1}^{2} \gamma_{2}^{2}}{c_{0}}\left(\gamma_{1}+2 \gamma_{2}\right)\right)
\end{gathered}
$$


Теперь найдем $\alpha_{10}+\alpha_{30}$ и $\alpha_{40}+\alpha_{20}$. Из (8) имеем тождества

$$
\begin{aligned}
& d_{10}-d_{30}=\alpha_{10}^{2}-\alpha_{30}^{2}+d_{11}\left(\alpha_{10}-\alpha_{30}\right)-\left(\alpha_{10}^{\prime}-\alpha_{30}^{\prime}\right), \\
& d_{20}-d_{40}=\alpha_{20}^{2}-\alpha_{40}^{2}+d_{21}\left(\alpha_{20}-\alpha_{40}\right)-\left(\alpha_{20}^{\prime}-\alpha_{40}^{\prime}\right) .
\end{aligned}
$$

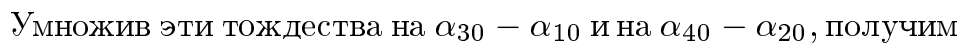

$$
\begin{aligned}
& G_{1}=\left(\alpha_{10}^{2}-\alpha_{30}^{2}\right)\left(\alpha_{30}-\alpha_{10}\right)-d_{11} F_{1}+\frac{1}{2} F_{1}^{\prime}, \\
& G_{2}=\left(\alpha_{20}^{2}-\alpha_{40}^{2}\right)\left(\alpha_{40}-\alpha_{20}\right)-d_{21} F_{2}+\frac{1}{2} F_{2}^{\prime},
\end{aligned}
$$

откуда

$$
\alpha_{10}+\alpha_{30}=\frac{\frac{1}{2} F_{1}^{\prime}-d_{11} F_{1}-G_{1}}{F_{1}}, \quad \alpha_{40}+\alpha_{20}=\frac{\frac{1}{2} F_{2}^{\prime}-d_{21} F_{2}-G_{2}}{F_{2}} .
$$

Следовательно,

$$
\begin{aligned}
& \alpha_{10}^{2}+\alpha_{30}^{2}=\frac{1}{2}\left(\alpha_{10}+\alpha_{30}\right)^{2}+\frac{1}{2}\left(\alpha_{10}-\alpha_{30}\right)^{2}=\frac{1}{2}\left(\frac{\frac{1}{2} F_{1}^{\prime}-d_{11} F_{1}-G_{1}}{F_{1}}\right)^{2}+\frac{1}{2} F_{1}, \\
& \alpha_{20}^{2}+\alpha_{40}^{2}=\frac{1}{2}\left(\alpha_{20}+\alpha_{40}\right)^{2}+\frac{1}{2}\left(\alpha_{20}-\alpha_{40}\right)^{2}=\frac{1}{2}\left(\frac{\frac{1}{2} F_{2}^{\prime}-d_{21} F_{2}-G_{2}}{F_{2}}\right)^{2}+\frac{1}{2} F_{2} .
\end{aligned}
$$

Из (8) выводим

$$
\begin{aligned}
& d_{10}+d_{30}=\alpha_{10}^{2}+\alpha_{30}^{2}-\left(\alpha_{10}^{\prime}+\alpha_{30}^{\prime}\right)+d_{11}\left(\alpha_{10}+\alpha_{30}\right), \\
& d_{20}+d_{40}=\alpha_{20}^{2}+\alpha_{40}^{2}-\left(\alpha_{20}^{\prime}+\alpha_{40}^{\prime}\right)+d_{21}\left(\alpha_{20}+\alpha_{40}\right) .
\end{aligned}
$$

Таким образом,

$$
\begin{aligned}
d_{10}+d_{30}= & \frac{1}{2}\left(\frac{\frac{1}{2} F_{1}^{\prime}-d_{11} F_{1}-G_{1}}{F_{1}}\right)^{2}+\frac{1}{2} F_{1}-\frac{d}{d x}\left(\frac{\frac{1}{2} F_{1}^{\prime}-d_{11} F_{1}-G_{1}}{F_{1}}\right) \\
& +d_{11}\left(\frac{\frac{1}{2} F_{1}^{\prime}-d_{11} F_{1}-G_{1}}{F_{1}}\right), \\
d_{20}+d_{40}= & \frac{1}{2}\left(\frac{\frac{1}{2} F_{2}^{\prime}-d_{21} F_{2}-G_{2}}{F_{2}}\right)^{2}+\frac{1}{2} F_{2}-\frac{d}{d x}\left(\frac{\frac{1}{2} F_{2}^{\prime}-d_{21} F_{2}-G_{2}}{F_{2}}\right) \\
& +d_{21}\left(\frac{\frac{1}{2} F_{2}^{\prime}-d_{21} F_{2}-G_{2}}{F_{2}}\right) .
\end{aligned}
$$

Воспользуемся формулами (20)-(23) и (11), чтобы выразить $\alpha_{10}+\alpha_{30}$ и $\alpha_{20}+\alpha_{40}$ через $\gamma_{1}$ и $\gamma_{2}$. Получим

$$
\begin{aligned}
H=\alpha_{10}+\alpha_{30} & =\frac{\frac{1}{2} F_{1}^{\prime}-d_{11} F_{1}-G_{1}}{F_{1}} \\
& =\frac{-2 \gamma_{2}^{2} \gamma_{1}^{\prime 2}+\gamma_{1}^{2}\left(2 \gamma_{1}^{\prime} \gamma_{2}^{\prime}-\gamma_{2} \gamma_{1}^{\prime \prime}\right)+\gamma_{1} \gamma_{2}\left(2 \gamma_{1}^{\prime 2}+\gamma_{2} \gamma_{1}^{\prime \prime}\right)}{\gamma_{1} \gamma_{2}\left(\gamma_{1}-\gamma_{2}\right) \gamma_{1}^{\prime}}, \\
\alpha_{20}+\alpha_{40} & =\frac{\frac{1}{2} F_{2}^{\prime}-d_{21} F_{2}-G_{2}}{F_{2}} \\
& =\frac{-2 \gamma_{1}^{2} \gamma_{2}^{\prime 2}+\gamma_{2}^{2}\left(2 \gamma_{1}^{\prime} \gamma_{2}^{\prime}-\gamma_{1} \gamma_{2}^{\prime \prime}\right)+\gamma_{1} \gamma_{2}\left(2 \gamma_{2}^{\prime 2}+\gamma_{1} \gamma_{2}^{\prime \prime}\right)}{\gamma_{1} \gamma_{2}\left(\gamma_{2}-\gamma_{1}\right) \gamma_{2}^{\prime}} .
\end{aligned}
$$


Отметим, что в случае выполнения равенства

$$
\alpha_{10}+\alpha_{30}=\alpha_{20}+\alpha_{40}
$$

уравнение (15) сильно упрощается и принимает вид:

$$
\frac{F_{1}}{2}-\frac{F_{2}}{2}=\frac{1}{\gamma_{1}}-\frac{1}{\gamma_{2}} .
$$

Имеем

$$
\alpha_{10}+\alpha_{30}-\left(\alpha_{20}+\alpha_{40}\right)=\frac{2 \gamma_{1}^{\prime} \gamma_{2}^{\prime}\left(\gamma_{1}^{\prime}+\gamma_{2}^{\prime}\right)+\left(\gamma_{1}-\gamma_{2}\right)\left(\gamma_{2}^{\prime \prime} \gamma_{1}^{\prime}-\gamma_{1}^{\prime \prime} \gamma_{2}^{\prime}\right)}{\left(\gamma_{1}-\gamma_{2}\right) \gamma_{1}^{\prime} \gamma_{2}^{\prime}}
$$

Как заметил М. В. Нешадим, равенство

$$
2 \gamma_{1}^{\prime} \gamma_{2}^{\prime}\left(\gamma_{1}^{\prime}+\gamma_{2}^{\prime}\right)+\left(\gamma_{1}-\gamma_{2}\right)\left(\gamma_{2}^{\prime \prime} \gamma_{1}^{\prime}-\gamma_{1}^{\prime \prime} \gamma_{2}^{\prime}\right)=0
$$

вытекает из

$$
A \gamma_{1} \gamma_{2}+B\left(\gamma_{1}+\gamma_{2}\right)-C=0, \quad A, B, C \in \mathbb{C} .
$$

Таким образом, если $\gamma_{2}$ выражается через $\gamma_{1}$ по формуле (4), то уравнение (15) принимает следующий вид:

$$
\frac{\gamma_{2}^{2} F\left(\gamma_{1}\right)}{2 c_{0} \gamma_{1}^{\prime 2}\left(\gamma_{1}-\gamma_{2}\right)^{2}}-\frac{\gamma_{1}^{2} F\left(\gamma_{2}\right)\left(B+A y_{1}\right)^{4}}{2 c_{0} \gamma_{1}^{\prime 2}\left(B^{2}+A C\right)^{2}\left(\gamma_{1}-\gamma_{2}\right)^{2}}=\frac{1}{\gamma_{1}}-\frac{1}{\gamma_{2}}
$$

тем самым оно интегрируемо в квадратурах (см. (5)). Теорема 1 доказана.

С помощью полученных формул найдем коэффициенты оператора шестого порядка. Из равенства

$$
\psi_{i}^{\prime \prime}=\chi_{0} \psi_{i}+\chi_{1} \psi_{i}^{\prime}
$$

выразим производные высокого порядка функции $\psi_{i}$ через $\psi_{i}, \psi_{i}^{\prime}, \chi_{0}$ и $\chi_{1}$ и подставим полученные выражения в

$$
\left(\partial_{x}^{6}+f_{5} \partial_{x}^{5}+f_{4} \partial_{x}^{4}+f_{3} \partial_{x}^{3}+f_{2} \partial_{x}^{2}+f_{1} \partial_{x}+f_{0}\right) \psi_{i}=\lambda \psi_{i}
$$

Получим

$$
P_{1} \psi_{i}+P_{2} \psi_{i}^{\prime}=\lambda \psi_{i}
$$

где

$$
\begin{aligned}
P_{1}= & \chi_{0}^{\mathrm{IV}}+4 \chi_{1}^{\prime \prime \prime} \chi_{0}+6 \chi_{1}^{\prime \prime} \chi_{0}^{\prime}+4 \chi_{1}^{\prime} \chi_{0}^{\prime \prime}+\chi_{1} \chi_{0}^{\prime \prime \prime}+4\left(\chi_{0}^{\prime}\right)^{2}+7 \chi_{0} \chi_{0}^{\prime \prime}+8\left(\chi_{1}^{\prime}\right)^{2} \chi_{0} \\
& +9 \chi_{1} \chi_{1}^{\prime \prime} \chi_{0}+7 \chi_{1} \chi_{1}^{\prime} \chi_{0}^{\prime}+\chi_{1}^{2} \chi_{0}^{\prime \prime}+6 \chi_{1}^{\prime} \chi_{0}^{2}+9 \chi_{1} \chi_{0} \chi_{0}^{\prime}+9 \chi_{1}^{\prime} \chi_{1}^{2} \chi_{0} \\
& +\chi_{1}^{3} \chi_{0}^{\prime}+\chi_{0}^{3}+3 \chi_{1}^{2} \chi_{0}^{2}+\chi_{1}^{4} \chi_{0}+f_{5}\left(\chi_{0}^{\prime \prime \prime}+3 \chi_{1}^{\prime \prime} \chi_{0}+3 \chi_{1}^{\prime} \chi_{0}^{\prime}+\chi_{1} \chi_{0}^{\prime \prime}+4 \chi_{0} \chi_{0}^{\prime}\right. \\
& \left.+5 \chi_{1} \chi_{1}^{\prime} \chi_{0}+\chi_{1}^{2} \chi_{0}^{\prime}+2 \chi_{1} \chi_{0}^{2}+\chi_{1}^{3} \chi_{0}\right) \\
& +f_{4}\left(\chi_{0}^{\prime \prime}+2 \chi_{1}^{\prime} \chi_{0}+\chi_{1} \chi_{0}^{\prime}+\chi_{0}^{2}+\chi_{1}^{2} \chi_{0}\right)+f_{3}\left(\chi_{0}^{\prime}+\chi_{1} \chi_{0}\right)+f_{2} \chi_{0}+f_{0}, \\
P_{2}= & 4 \chi_{0}^{\prime \prime \prime}+7 \chi_{1}^{\prime \prime} \chi_{0}+12 \chi_{1}^{\prime} \chi_{0}^{\prime}+9 \chi_{1} \chi_{0}^{\prime \prime}+6 \chi_{0} \chi_{0}^{\prime}+15 \chi_{1}^{\prime} \chi_{1} \chi_{0}+9 \chi_{1}^{2} \chi_{0}^{\prime} \\
& +3 \chi_{1} \chi_{0}^{2}+4 \chi_{1}^{3} \chi_{0}+\chi_{1}^{\mathrm{IV}}+10 \chi_{1}^{\prime \prime} \chi_{1}^{\prime}+5 \chi_{1} \chi_{1}^{\prime \prime \prime}+15\left(\chi_{1}^{\prime}\right)^{2} \chi_{1} \\
& +10 \chi_{1}^{\prime} \chi_{1}^{3}+10 \chi_{1}^{\prime \prime} \chi_{1}^{2}+\chi_{1}^{5}+f_{5}\left(3 \chi_{0}^{\prime \prime}+4 \chi_{1}^{\prime} \chi_{0}+5 \chi_{1} \chi_{0}^{\prime}+\chi_{0}^{2}+3 \chi_{1}^{2} \chi_{0}\right. \\
& \left.+\chi_{1}^{\prime \prime \prime}+3\left(\chi_{1}^{\prime}\right)^{2}+4 \chi_{1} \chi_{1}^{\prime \prime}+6 \chi_{1}^{\prime} \chi_{1}^{2}+\chi_{1}^{4}\right) \\
& +f_{4}\left(2 \chi_{0}^{\prime}+2 \chi_{1} \chi_{0}+\chi_{1}^{\prime \prime}+3 \chi_{1} \chi_{1}^{\prime}+\chi_{1}^{3}\right)+f_{3}\left(\chi_{0}+\chi_{1}^{\prime}+\chi_{1}^{2}\right)+f_{2} \chi_{1}+f_{1} .
\end{aligned}
$$

Из равенства $(2)$ и из того, что $\chi_{0}$ и $\chi_{1}$ не зависят от выбора $x_{0}$, следует

$$
P_{1}=\lambda, \quad P_{2}=0 .
$$

Коэффициенты $f_{j}$ находятся из разложений $\chi_{i}$ и $\lambda$ в ряд по степеням $z$ в окрестности $q$. 


\section{Список литературы}

1. Burchnall J. L., Chaundy I. W. Commutative ordinary differential operators // Proc. London Math. Soc. Ser. 2. 1923. V. 21. P. 420-440.

2. Burchnall J. L., Chaundy I. W. Commutative ordinary differential operators // Proc. Royal Soc. London Ser. A. 1928. V. 118. P. 557-583.

3. Burchnall J. L., Chaundy I. W. Commutative ordinary differential operators // Proc. Royal Soc. London Ser. A. 1931. V. 134. P. 471-485.

4. Кричевер И. М. Интегрирование нелинейных уравнений методами алгебраической геометрии // Функц. анализ и его прилож. 1977. Т. 11. №1. С. 15-31.

5. Кричевер И. М. Коммутативные кольца линейных обыкновенных дифференциальных операторов // Функц. анализ и его прилож. 1978. Т. 12. № 4. С. 41-52.

6. Кричевер И. М., Новиков С. П. Голоморфные расслоения над римановьпи поверхностями и уравнение Кадомцева-Петвиашвили. I // Функц. анализ и его прилож. 1978. Т. 12 . № 4. C. 41-52.

7. Кричевер И. М., Новиков С. П. Голоморфные расслоения над алгебраическими кривьми и нелинейные уравнения // УМН. 1980. Т. 35. №6. С. 47-68.

8. Мохов О. И. Коммутирующие дифференциальные операторы ранга 3 и нелинейные уравнения // Изв. АН СССР. Сер. матем. 1989. Т. 53. №6. С. 1291-1314.

9. Dehornoy P. Operateurs differentiels et courbes elliptiques // Compositio Math. 1981. V. 43. № 1. P. 71-99.

10. Новиков С. П., Гриневич П. Г. О спектральной теории коммутирующих операторов ранга 2 с периодическими коэффициентами // Функц. анализ и его прилож. 1982. Т. 16. № 1. C. $25-26$.

11. Новиков С. П. Коммутирующие операторы ранга $l>1$ с периодическими коэффицциентами // Докл. АН СССР. 1982. Т. 263. №6. С. 1311-1313.

12. Диксмье ЖК. Об алгебрах Вейля // Математика. 1969. Т. 13. № 4. С. 16-44.

13. Гриневич П. Г. Рациональные решения уравнений коммутации дифференциальных операторов // Функц. анализ и его прилож. 1982. Т. 16. №1. С. 19-24.

Институт математики

им. С. Л. Соболева СО РАН, г. Новосибирск

Поступила в редакцию

E-mail: mironov@ngs.ru

05.06.2003 